\title{
Hepatite C: prevenção e diagnóstico
}

\author{
Josidel Conceição OLIVER $^{1}$ \\ Clara Gavião PRADO ${ }^{1}$ \\ Cristiane Carneiro OLIVEIRA ${ }^{1}$ \\ Dalila Junqueira ALVARENGA ${ }^{1}$ \\ Deliza Rodrigues Santos Alves da COSTA ${ }^{1}$ \\ Jaqueline de Lima GERMANO ${ }^{1}$ \\ Jéssica Maria NERY ${ }^{1}$ \\ Joyce Oliveira NERY ${ }^{1}$ \\ Juliane Naiara FERREIRA ${ }^{1}$ \\ Larissa Ferreira SELICANI ${ }^{1}$ \\ Larissa Rocha Arruda de SOUZA ${ }^{1}$ \\ Lênin Machado ROSA ${ }^{1}$ \\ Lília de Oliveira MONTEIRO ${ }^{1}$ \\ Nadielle Gonçalves SIQUEIRA ${ }^{1}$ \\ Sandra Maria Oliveira Morais VEIGA ${ }^{2}$
}

\section{Recebido em: 30/05/2013 - Aprovado em: 12/07/2013 - Disponibilizado em: 15/08/2013}

RESUMO: A hepatite C é uma doença causada pelo vírus da hepatite C (HCV), transmitida via sanguínea, crônica e silenciosa que acomete cerca de $3 \%$ da população mundial. As manicures podem contribuir para a disseminação da doença. Este estudo objetivou multiplicar informações sobre a Hepatite $\mathrm{C}$, riscos inerentes à profissão, medidas de prevenção e de segurança no trabalho para as manicures e do município de Alfenas-MG. Foram ministrados dois cursos interativos, abrangendo 30 profissionais, visando esclarecê-las sobre o assunto, enfatizando os fatores de risco, importância da proteção da sua saúde e de suas clientes e do diagnóstico precoce. Foram distribuídos questionários no início do curso, para avaliar o conhecimento prévio. Ao final, realizou-se um debate para verificar o aprendizado das participantes. Ainda, disponibilizou-se o teste sorológico para a detecção de hepatite C e suporte ambulatorial, caso necessário. Observou-se que $93 \%$ das manicures sabiam que a doença pode ser transmitida pelo sangue e $86 \%$, nunca haviam sido orientadas sobre os cuidados com os instrumentos perfuro-cortantes. Dos 30 testes sorológicos realizados, todos foram 'soro não reagente'. Observou-se ainda, que as manicures são resistentes à implantação de práticas preventivas, principalmente, em função do custo adicional. As manicures foram esclarecidas quanto à necessidade de se implantar ações preventivas para reduzir o risco potencial de transmissão da doença em seu ambiente de trabalho.

Palavras-Chave: HCV. Manicures. Perfuro-cortantes. Sorologia. Hepatites.

\section{Hepatitis C: prevention e diagnostic}

\begin{abstract}
Hepatitis $\mathrm{C}$ is a disease caused by the hepatitis $\mathrm{C}$ virus (HCV), transmitted via blood, chronic and silent that affects about $3 \%$ of world population. The manicures can contribute to the spread of the disease. This study aimed

\footnotetext{
${ }^{1}$ Graduandos/Graduados em Farmácia, Bolsistas do Programa de Educação Tutorial, Grupo PET-Farmácia, Faculdade de Ciências Farmacêuticas, Universidade Federal de Alfenas, UNIFAL - MG. E-mail: petfarmaciaunifal@googlegroups.com.

2 Profa Dra em Ciência dos Alimentos e Tutora do Programa de Educação Tutorial de Farmácia, Faculdade de Ciências Farmacêuticas, Universidade Federal de Alfenas, UNIFAL - MG. E-mail: smveiga@gmail.com.
} 
to multiply information about hepatitis $\mathrm{C}$ risks inherent to the profession, prevention and safety at work for manicures and Alfenas-MG. Two interactive courses were given to 30 professionals, aiming to enlighten them on the subject, emphasizing the risk factors, the importance of protecting your health and your customers and early diagnosis. Questionnaires were distributed at the beginning of the course to assess prior knowledge. At the end, there was a debate to check the learning of the participants. Also, serological tests for the detection of hepatitis $\mathrm{C}$ were made available and, if necessary, ambulatory support. It was observed that $93 \%$ of manicures knew that the disease can be transmitted by blood and $86 \%$ had never been instructed on the care of the skin-piercing instruments. It was performed 30 serological tests and all were serum non reactive. It was also observed that the manicures are resistant to the implementation of preventive practices, mainly due to the additional cost. The manicures were enlightened on the need to implement preventive actions to reduce the potential risk of disease transmission in the workplace.

Keywords: HCV. Manicures. Sharps instruments. Serology. Hepatitis.

\section{INTRODUÇÃO}

Grave problema de saúde pública no Brasil e no mundo, a hepatite é a inflamação do fígado, que pode ser causada por vírus, uso de alguns medicamentos, álcool e outras drogas, além de doenças autoimunes, metabólicas e genéticas. É uma doença silenciosa que nem sempre apresenta sintomas, mas quando estes aparecem, podem ser detectados: cansaço, febre, mal-estar, tontura, enjoo, vômitos, dor abdominal, pele e olhos amarelados, urina escura e fezes claras (BRASIL, 2012a; BRASIL 2008).

Estima-se que cerca de 170 milhões de pessoas encontravam-se infectadas de forma crônica pelo HCV em 2011 e que ocorrem 350 mil mortes causadas por esta doença a cada ano, no mundo (WORLD HEALTH ORGANIZATION, 2012a).

No Brasil, as hepatites virais mais comuns são as causadas pelos vírus A, B e C, sendo que milhões de pessoas são portadoras dos vírus $\mathrm{B}$ ou $\mathrm{C}$ e não sabem (BRASIL, 2008; BRASIL, 2012a).
As hepatites virais são doenças de notificação compulsória, ou seja, cada ocorrência deve ser notificada por um profissional de saúde. Esse registro é importante para mapear os casos de hepatites no país e ajudar a traçar diretrizes de políticas públicas no setor (BRASIL, 2008; BRASIL, 2012a).

Entre as formas de transmissão das hepatites "B" e "C" estão: transfusão de sangue; compartilhamento de material para uso de drogas (seringas, agulhas, cachimbos, entre outros), artigos para higiene pessoal (lâminas de barbear e depilar, escovas de dente, alicates de unha ou outros objetos que furam ou cortam) ou para confecção de tatuagem e colocação de piercings; ainda, a transmissão congênita e sexo sem o uso de preservativos (BRASIL, 2008; BRASIL, 2012a).

A forma aguda da hepatite $\mathrm{C}$ é geralmente assintomática e, portanto, de difícil detecção. Em 70 a $85 \%$ dos casos ocorre a cronificação, podendo evoluir para as formas histológicas graves como cirrose e carcinoma hepático. Nessa fase, o tratamento 
é difícil e complexo, o que enfatiza a importância do diagnóstico e tratamento precoces da doença (BRASIL, 2008).

Devido às formas de transmissão da hepatite $\mathrm{C}$, alguns segmentos da população estão mais sujeitos ao contato com o $\mathrm{HCV}$; as manicures correm sérios riscos de contaminação e também podem disseminar a doença por meio dos seus instrumentos de trabalho (alicates, espátulas, palitos e lixas de unha), quando não dispensam os cuidados higiênicos necessários (esterilização e uso individual).

Diante do exposto, faz-se necessário o desenvolvimento de ações e projetos voltados para os segmentos mais expostos ao risco de contrair a doença e para a população em geral. A informação e orientação das pessoas quanto às formas de transmissão, prevenção, diagnóstico precoce e tratamento adequado da doença, podem reduzir significativamente a disseminação e as complicações da Hepatite C.

\section{METODOLOGIA}

\subsection{Confecção de folders educativos.}

No intuito de facilitar o processo ensino-aprendizagem, foram elaborados folders de caráter educativo, incluindo aspectos relacionados às hepatites virais, com ilustrações e informações que despertam ainda mais o interesse do público pelo assunto. Estes folders foram distribuídos nos cursos, palestras, eventos e demais atividades que o grupo PET-FARMÁCIA da UNIFALMG organizou/participou nos últimos dois anos.

\subsection{Cadastro das manicures do município de Alfenas.}

Inicialmente, as manicures foram convidadas em seus locais de trabalho para participar de cursos de capacitação para prevenção da transmissão da Hepatite $\mathrm{C}$ em sua atividade. Os encontros foram realizados na UNIFAL-MG e em uma escola profissionalizante local.

\subsection{Aplicação do questionário sobre conhecimentos prévios sobre o tema.}

Antes de iniciar os cursos sobre hepatite, as manicures foram questionadas em relação à prevenção e diagnóstico de hepatite $\mathrm{C}$, testando seus conhecimentos prévios sobre o assunto por meio da utilização de um questionário simples, previamente elaborado (FIGURA 1).

\subsection{Palestras e cursos para manicures.}

As palestras e os cursos foram realizados com a explanação do tema, apresentação de slides/ vídeos / DVD e abertura para discussão e esclarecimento de dúvidas sobre o assunto em qualquer 
momento da atividade. Assim, foram empregadas técnicas pedagógicas expositivas (palestras) ou interativas (oficinas, grupos de discussão / dinâmica de grupo), conforme tempo disponibilizado pela instituição parceira. Foram distribuídos ainda, os folders previamente elaborados.

FIGURA 1 - Questionário sobre Hepatite C.

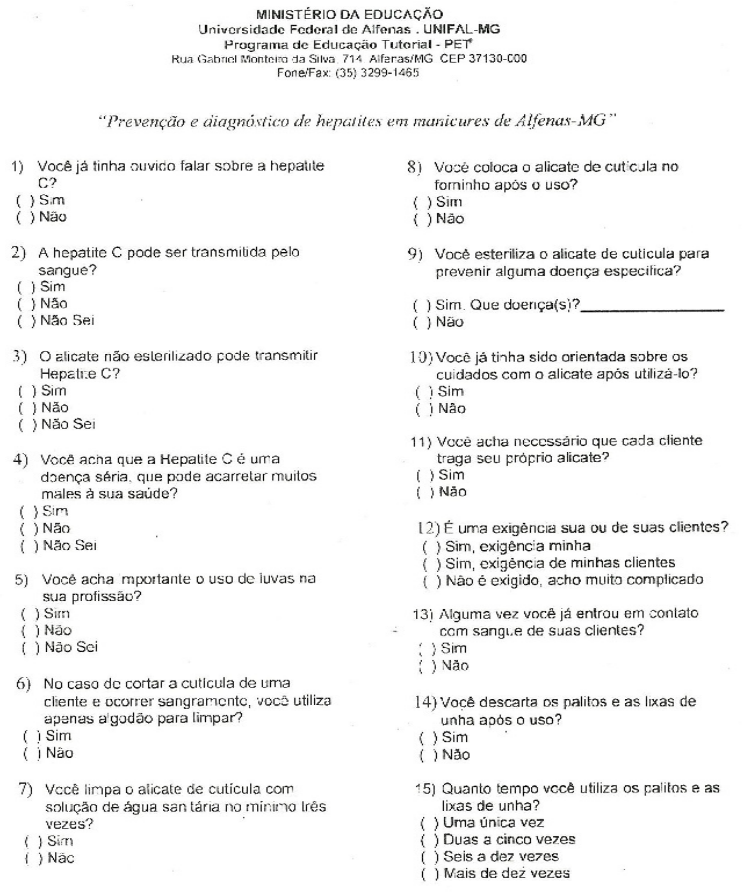

Nas oficinas educativas, as manicures foram treinadas para desenvolver técnicas adequadas para a limpeza e esterilização do instrumental de trabalho.

Os assuntos abordados abrangeram: conhecimentos gerais sobre hepatites virais, bem como suas formas de contaminação e transmissão, complicações da doença e a importância da prevenção da mesma. Um enfoque maior foi dado à hepatite $\mathrm{C}$ e os cuidados que as manicures devem ter para evitar o contato e a disseminação do vírus em seu ambiente de trabalho.

Por fim, foi explicado o procedimento adotado para realização do teste sorológico rápido para hepatite $\mathrm{C}$, esclarecido o conceito de janela imunológica e nos casos de interesse das manicures, foi disponibilizado o teste rápido para hepatite $\mathrm{C}$, com assinatura prévia dos termos de consentimento (FIGURA 2).

ANEXO 2 - Termo de consentimento para realização do teste.

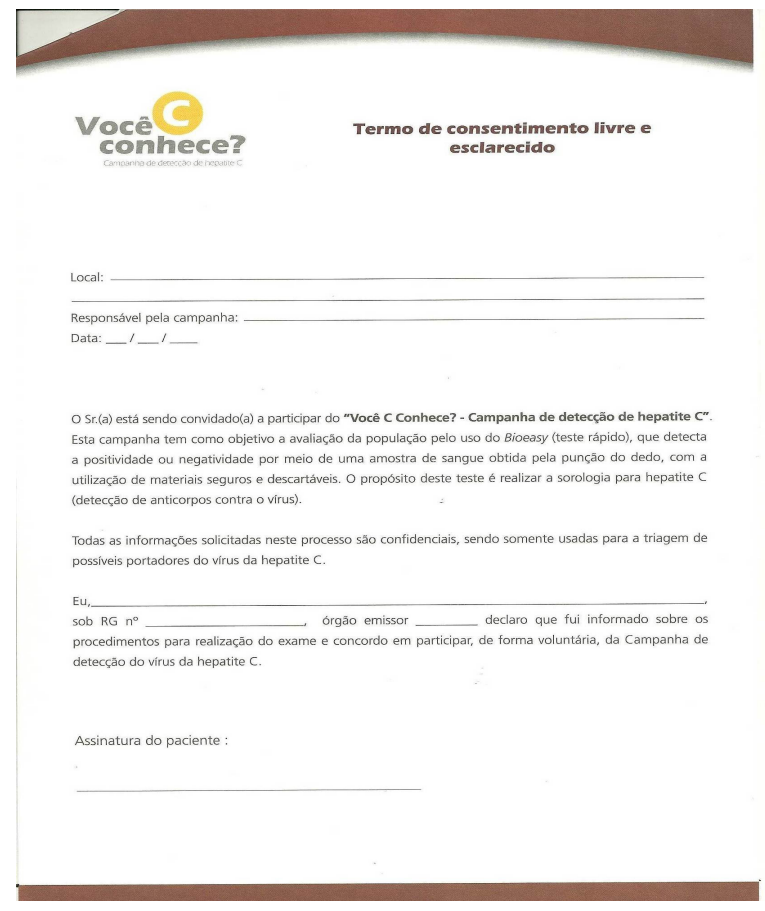

No caso de sorologia reagente, o trabalhou teve como parceiro o Centro de Testagem e Aconselhamento (CTA) de Alfenas-MG e assim, as pacientes podiam ser encaminhadas para sorologia confirmatória e 
se necessário, tratamento e acompanhamento médico, psíquico e social.

\subsection{Palestras educativas para outros públicos.}

Foram realizadas palestras sobre prevenção e diagnóstico de hepatite $\mathrm{C}$ para a população em geral, abrangendo diferentes faixas etárias e grupos socioculturais. Os assuntos abordados foram hepatites virais, suas formas de transmissão, complicações e a importância da prevenção e do diagnóstico precoce da doença.

Também foi explicado o procedimento adotado para realização do teste sorológico rápido para hepatite $\mathrm{C}$ e este foi demonstrado, como forma de estimular a sorologia voluntária, que pôde ser realizada gratuitamente em muitos eventos que o Grupo PET-Farmácia da UNIFAL-MG participou (Campanha nacional de prevenção e controle da Hepatite C, COOPFAM, Semana Nacional de Ciência e Tecnologia) ou no CTA de Alfenas, com todo acompanhamento necessário. O conceito de janela imunológica também foi esclarecido. $\mathrm{E}$ os folders previamente elaborados foram distribuídos. Toda a atividade foi aberta ao esclarecimento de dúvidas sobre o assunto.

\subsection{Teste rápido Anti-HCV}

Antes da realização do teste, as manicures e demais participantes assinaram um termo de consentimento de participação voluntária para realização do teste (FIGURA 2) e foram entrevistados sobre características sociodemográficas (idade, sexo, estado civil, escolaridade, profissão, raça/cor) e possíveis fatores de risco para infecção pelo HCV (uso de drogas, história de transfusão de sangue e de cirurgia, tatuagem/piercing, uso de preservativo, número de parceiros sexuais, história de doença sexualmente transmissível). Além disso, foram questionados quanto à realização ou não do teste em questão (FIGURA 3).

FIGURA 3 - Formulário de Aconselhamento Pré-teste.

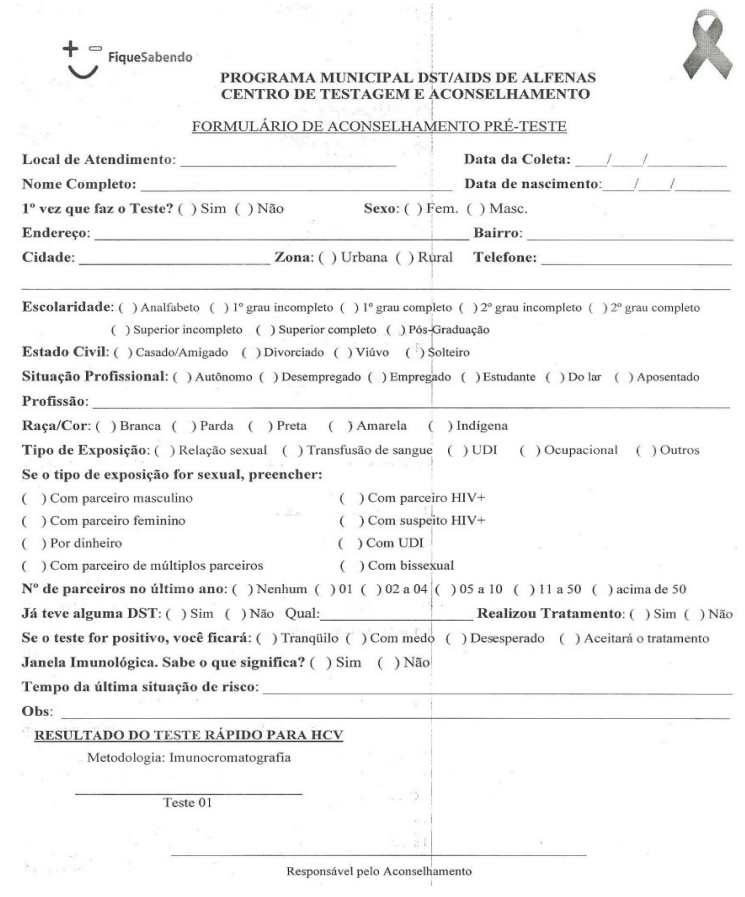

Em seguida, o teste foi realizado de acordo com as especificações dos fabricantes dos reagentes.

Para isto, o dispositivo foi removido da embalagem. Em seguida, foi coletada de 
uma amostra de sangue do(a) voluntário(a) com lanceta e capilar e transferido para cavidade "S" do dispositivo de teste. Quatro gotas do tampão diluente foram adicionadas em tal cavidade e o resultado foi interpretado entre 15 e 20 minutos (HCV RAPID TEST BIOEASY, 2012).

O resultado foi interpretado como não reagente quando apresentou somente uma linha colorida na janela de resultados, a linha de controle "C", sendo que esta linha deve estar presente em todos os testes (HCV RAPID TEST BIOEASY, 2012).

A interpretação de teste reagente ocorreu quando foram observadas duas linhas coloridas na janela de resultados, a linha de controle "C" e a linha teste "T". A intensidade das duas linhas pode ser diferente, porém o resultado é considerado reagente (HCV RAPID TEST BIOEASY, 2012).

O resultado foi considerado inválido quando nenhuma linha colorida apareceu na janela de resultados dentro de 20 minutos ou quando a linha controle "C" não apareceu na janela de resultados dentro de 20 minutos (HCV RAPID TEST BIOEASY, 2012).

Concluído o teste, foi preenchida uma ficha com o resultado obtido na análise, a qual foi entregue ao voluntário com as necessárias orientações (FIGURAS 4 e 5).

O teste realizado consiste em uma triagem para a infecção pelo $\mathrm{HCV}$, sendo considerado um diagnóstico sorológico inicial. A presença de anti-HCV não define isoladamente a presença de infecção ativa e deve ser interpretada como contato prévio com o HCV. O resultado reagente desse marcador deve ser confirmado por testes moleculares para detecção de ácidos nucléicos do HCV (HCV RAPID TEST BIOEASY, 2012).

FIGURA 4 - Laudo de Triagem - Soro Reagente

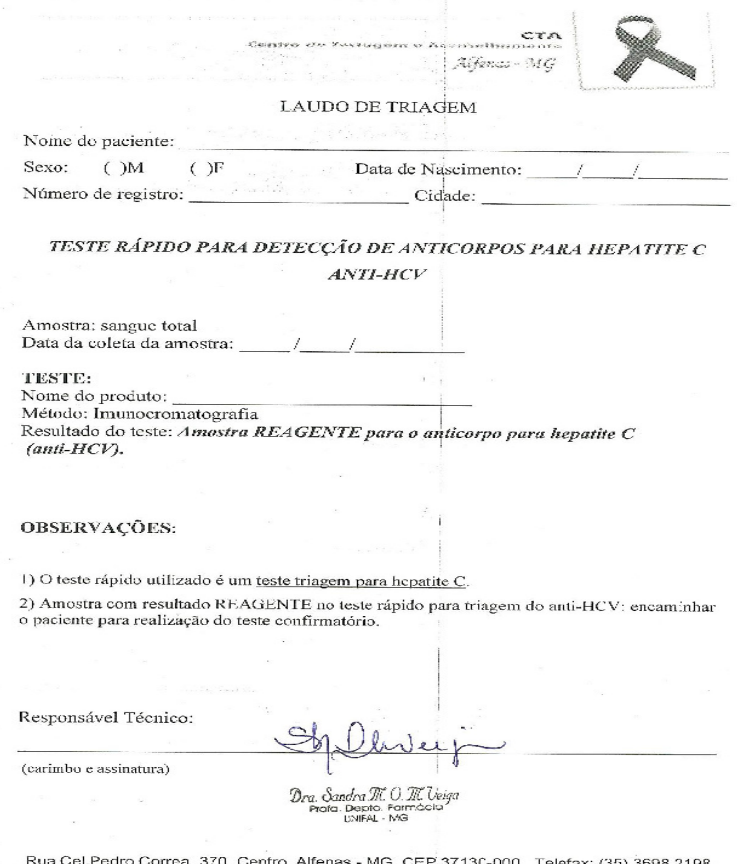


FIGURA 5 - Laudo de Triagem - Soro Não Reagente

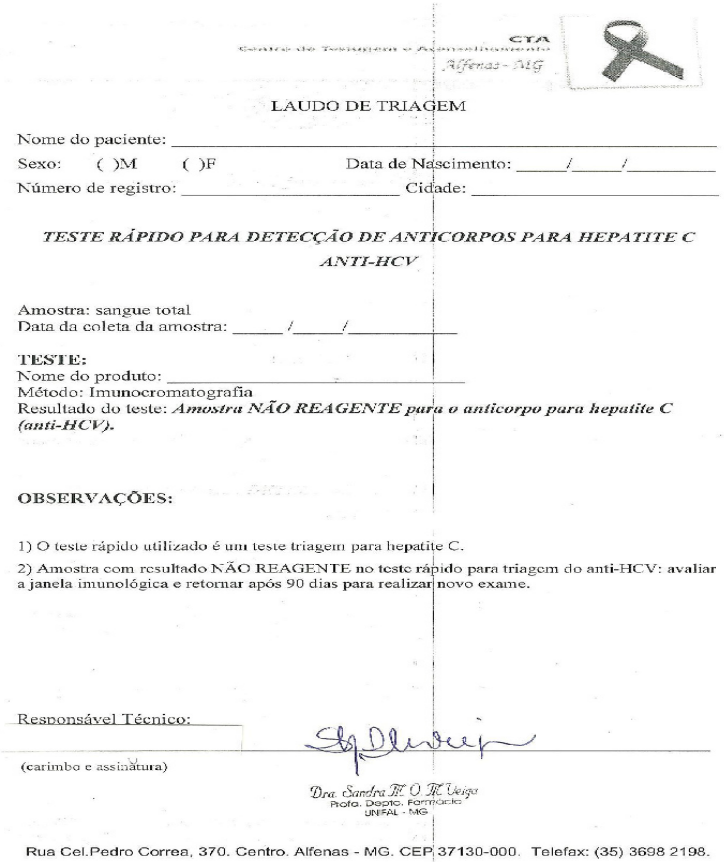

As pessoas que apresentaram resultado soro reagente, neste estudo, foram encaminhadas ao Centro de Testagem e Aconselhamento (CTA) da cidade de Alfenas para sorologia confirmatória e acompanhamento necessário.

\section{RESULTADOS E DISCUSSÃO}

Os dados obtidos foram tabulados e determinaram-se as frequências relativas para as diferentes respostas obtidas nas entrevistas com as manicures.

\subsection{Manicures}

Observou-se que $93,1 \%$ das manicures sabiam que a hepatite pode ser transmitida pelo sangue (TABELA 1) e 86,2\% delas nunca haviam sido orientadas sobre os cuidados que devem ser dispensados aos instrumentos perfuro cortantes empregados em sua rotina de trabalho. Em um estudo realizado em São Paulo, com manicures e/ou pedicures, foi observado que $85 \%$ sabiam como ocorre a transmissão da hepatite $\mathrm{C}$ e 95\% não sabiam como evitá-la (OLIVEIRA; FOCACCIA, 2010).

Ainda, por meio das informações obtidas no referido questionário, verificou-se que $96,5 \%$ das entrevistadas acham que é importante o uso de luvas na profissão. Em uma pesquisa realizada em salões de beleza de São Paulo, com 100 manicures e/ou pedicures, $80 \%$ disseram não utilizar luvas cirúrgicas durante os procedimentos, apesar do fato de a maioria (84\%) dessas profissionais saberem que as luvas são instrumento para a sua proteção, e para a proteção dos seus clientes. Entre os $20 \%$ que declararam usar luvas, apenas $5 \%$ foram observadas utilizando este Equipamento de Proteção Individual (EPI) (OLIVEIRA; FOCACCIA, 2010).

Tabela 1 - Respostas das manicures às perguntas do questionário.

\begin{tabular}{llllll}
\hline Perguntas & & SIM & NÃO & $\begin{array}{l}\text { NÃO } \\
\text { SEI }\end{array}$ \\
\hline A hepatite C pode ser & $93,1 \%$ & $0 \%$ & $6,9 \%$ \\
transmitida pelo sangue? & & & \\
O alicate não esterilizado & $100 \%$ & $0 \%$ & $0 \%$
\end{tabular}


pode transmitir hepatite $\mathrm{C}$ ?

Você acha que a hepatite C é $\quad 96,5 \% \quad 0 \% \quad 3,5 \%$ uma doença séria?

Todas as manicures participantes do estudo relataram que o ideal seria que cada cliente trouxesse seu próprio alicate.

Atualmente, sabe-se que, para a prevenção de infecções em salões de manicure, cada cliente deve ter seu kit próprio de manicure (alicate, espátula e palito) e também seu esmalte, pois muitos fungos podem sobreviver no mesmo (SBCD, 2013).

Dos 30 testes sorológicos realizados, $100 \%$ dos resultados obtidos foram 'soro não reagente', embora metade $(50 \%)$ das manicures já tenha entrado em contato com sangue das clientes. Em um estudo com 82 manicures foi encontrado índice semelhante a este estudo com resultado anti-HCV não reagente para todas as manicures (MEZZOMO et al., 2011). Em uma pesquisa realizada em salões de beleza de São Paulo, com 100 manicures e/ou pedicures, dois por cento delas foram diagnosticadas como portadores do vírus da hepatite $\mathrm{C}$ (OLIVEIRA; FOCACCIA, 2010).

De acordo com os dados obtidos, é possível observar que ainda persiste desconhecimento acerca das formas de transmissão da doença entre as manicures. Também foi possível perceber que, mesmo sabendo de certos riscos aos quais estas profissionais estão expostas, as manicures ainda são resistentes ao uso de luvas e outras práticas preventivas, principalmente em função dos custos adicionais.

\subsection{Demais públicos}

Foi realizada uma campanha para prevenção e detecção de Hepatite C nos Postos de Saúde e Programa de Saúde da Família (PSF) da cidade de Alfenas, MG, nos dias 8 a 12 de agosto de 2011. O evento destinou-se a toda população da cidade de Alfenas - MG, sendo organizado pela Prefeitura Municipal de Alfenas, por meio do CTA, tendo a parceria do grupo PET Farmácia UNIFAL-MG. Orientou-se a população sobre as formas de contágio e transmissão da doença, bem como sobre as medidas preventivas e de controle. Foram aplicados questionários próprios da Campanha e realizados 2000 testes rápidos para hepatite $\mathrm{C}$, dos quais foram detectados 7 casos 'soro reagente', com incidência de 0,35\%. Estes foram encaminhados ao CTA para sorologia confirmatória e acompanhamento especializado.

Durante a Semana Nacional de Ciência e Tecnologia, o grupo participou do evento na COOPFAM, em Poço Fundo, sendo que 150 pessoas foram informadas sobre Hepatites Virais e fizeram testes rápidos de detecção da hepatite $\mathrm{C}$, sendo todos os testes 'soro não reagente'. 
Em um estudo realizado com uma população de gestantes foi encontrado positividade para sorologia anti-HCV em 0,11\% das pacientes (Pinto et al., 2011). Em um estudo realizado em ribeirinhos da Amazônia brasileira o marcador serológico para a hepatite C (HCV IgG específica) foi reativo em 8,8\% (16/181) dos indivíduos (OLIVEIRA et al., 2011). Uma investigação epidemiológica realizada entre as populações ribeirinhas e de imigrantes na Amazônia brasileira indicou positividade para sorologia anti-HCV variando entre de $0.38 \%$ (KHOURI et al., 2005), 1.7\% (Paula et al., 2001), 4.2\% (Tavares-Neto et al., 2004) e 4,8\% (PARANÁ et al., 2007). Estes estudos anteriormente citados, mostram altas taxas de incidência na positividade de sorologia anti-HCV na Região Norte do país. Entretanto, segundo Brasil (2012b), no período de 1999 a 2011, foram notificados 82.041 casos de hepatite $\mathrm{C}$ no Brasil, a maioria nas Regiões Sudeste $(67,3 \%)$ e Sul (22,3\%), e apenas (2,0\%) dos casos no Norte. A maior incidência nas regiões Sul e Sudeste pode estar relacionada à concentração populacional nessas regiões e ainda, a facilidade de acesso aos serviços de saúde, ao diagnóstico e profissionais de saúde capacitados para a notificação dos casos detectados.

O grupo participou ainda, de 06 oficinas educativas para 200 alunos de $7^{\circ}$ ao $9^{\circ}$ anos da Escola Municipal Orlando Paulino da Costa (zona rural de Alfenas, MG) e 02 palestras para cerca de 200 alunos de $6^{\circ}$ ao $9^{\circ}$ ano da FUNDAMAR (Fazenda Escola localizada no município de Paraguaçu, no sul de Minas, que atende crianças e jovens em situação de risco). Ao todo, trabalhou-se com 600 alunos do ensino fundamental para multiplicação de conhecimento sobre Hepatites Virais.

\section{CONCLUSÃO}

Foram realizadas ações educativas e a sorologia para a hepatite $\mathrm{C}$ para manicures e para a população em geral.

Observou-se que as manicures são resistentes a implantação de práticas preventivas, mesmo sabendo dos riscos, principalmente em função do custo adicional. As manicures foram alertadas quanto à necessidade de ações preventivas para reduzir o risco de transmissão da doença em seu ambiente de trabalho.

Nas atividades educativas realizadas para a população em geral, verificou-se grande interesse e participação dos diferentes segmentos (adolescentes, jovens, adultos e idosos).

Os resultados alcançados estimulam a proposição de novos cursos, oficinas e atividades educativas pelo Grupo PETFarmácia da Unifal-MG. 


\section{REFERÊNCIAS}

BRASIL. Ministério da Saúde. Secretaria de Políticas de Saúde. Programa Nacional de Hepatites Virais. Hepatites Virais: o Brasil está atento. Brasília: Ministério da Saúde, 2008.

BRASIL. Ministério da Saúde. HEPATITES

VIRAIS. Departamento de DST, Aids e Hepatites Virais. O que são hepatites?

Disponível em:

$<$ http://www.aids.gov.br/pagina/o-que-saohepatites-virais $>$. Acesso em: 14 de maio de 2012a.

BRASIL. Ministério da Saúde - Secretaria de Vigilância em Saúde - Departamento de DST, Aids e Hepatites Virais. Boletim

Epidemiológico - Hepatites Virais. Ano III n. 1, 2012a.

HCV RAPID TEST - Bioeasy I bioeasy, biomolecular, point of care, elisa, uroanálise. Disponível em:

$<$ http://www.bioeasy.com.br/site/produto.php ?n=40>. Acesso em 28 de abril de 2012.

KHOURI, M. E. L.; DUARTE, L. S.; RIBEIRO, R. B.; SILVA, L. F. F.; CAMARGO, L. M. A.; SANTOS, V. A.; BURATTINI, M. N.; CORBETT, C. E. P. Seroprevalence of hepatitis $\mathrm{B}$ virus and hepatitis $\mathrm{C}$ virus in Monte Negro in the Brazilian Western Amazon Region. Clinics. v. 60, n. 1, p. 29-36, Jan./Feb. 2005.

MEZZOMO, J.; SANTANA, A. O. T. O.; STRÖHER, D.; ZURAVSKI, L.; PILAR, B. C.; PAVIN, N. F.; FRANDALOZ, D. C.; BITTENCOURT, R.; MANFREDINI, V.; MANFREDINE, V. Inquérito sorológico para hepatites $\mathrm{B}$ e $\mathrm{C}$ em manicures e pedicures no Município de Uruguaiana - RS. Anais do Salão Internacional de Ensino, Pesquisa e
Extensão. Disponível em:

http://seer.unipampa.edu.br/index.php/siepe/a rticle/view/3465. Acesso em: 18 de maio de 2013. v. 3, n. 2, 2011.

OLIVEIRA, A. C. D. S. FOCACCIA, R. Survey of hepatitis B and C infection control: procedures at manicure and pedicure facilities in São Paulo, Brazil. Brazilian Journal of Infectious Diseases. v. 14, n. 5, p.502-507, 2010 .

OLIVEIRA, C. S. F.; SILVA, A. V.; SANTOS, K. N.; FECURY, A. A.; ALMEIDA, M. K. C.; FERNANDES, A. P.; COSTA, C. A.; FREITAS, A. S.; CORVELO, T. C. O.; MARTINS, L. C. Infecção pelo virus da hepatite $\mathrm{B}$ e $\mathrm{C}$ em ribeirinhos da Amazônia brasileira. Revista da Sociedade Brasileira de Medicina Tropical. v. 44, n. 5, Uberaba, Sept./Oct. 2011.

PARANÁ, R.; PAIVA, T.; LEITE, M. R.; OLIVEIRA, F. N.; KALI, N.; LOBATO, C.; DANTAS, T.; NETO, J. T. Infection with hepatitis $\mathrm{C}$ virus amongst health workers in the brazilian western Amazon Region (Rio Branco, State of Acre). The American Journal of Tropical Medicine and Hygiene. v. 76, n, 1, p. 165-169, 2007.

PAULA VS, ARRUDA ME, VITRAL CL, GASPAR AMC. Seroprevalence of Viral Hepatitis in Riverine Communities from the Western Region of the Brazilian Amazon Basin. Memórias do Instituto Oswaldo

Cruz. v. 96, n. 8, p. 1123-1128, 2001.

PINTO, C. S; MARTINS, R. M. B.; ANDRADE, S. M. O.; STIEF, A. C. F.; OLIVEIRA, R. D.; CASTRO, A. R. C. M. Infecção pelo vírus da hepatite $\mathrm{C}$ em gestantes em Mato Grosso do Sul, 2005-2007. Revista de Saúde Pública. v. 45, n. 5, p. 974-976, 2011. 
SBCD. Sociedade Brasileira de Cirurgia

Dermatológica. Química de esmaltes não

mata fungos, diz pesquisa em Rio Preto.

São Paulo: SBCD, 2013. Disponível em:

http://www.sbcd.org.br/noticia/2511

Acesso em: 30/05/2013.

TAVARES-NETO, J.; ALMEIDA, D.;

SOARES, M. C.; UCHOA, R.; VIANA, S.;

DARUB, R.; FARIAS E.; ROCHA, G.;

VITVITSKI, L.; PARANÁ, R.

Seroprevalence of hepatitis B and C in the

Western Brazilian Amazon region (Rio

Branco, Acre): a pilot study carried out during

a hepatitis B vaccination program. Brazilian

Journal of Infectious Diseases. v.8, n. 2,

Salvador Apr. 2004.

http://dx.doi.org/10.1590/S1413-

86702004000200003

WORLD HEALTH ORGANIZATION.

Hepatitis C. Genebra, 2012. Disponível em:

<http://www.who.int/mediacentre/factsheets/f

s164/en/>. Acesso em: 11 mar. 2012. 